\title{
Sociodemographic and family correlates of dietary-lifestyle patterns in young men: cross-sectional study (MeDiSH Project)
}

\section{Abstract}

The aim of this study was to identify the sociodemographic and family correlates associated with dietary and lifestyle patterns in young men. In all, 367 men from the Warmia and Mazury region (Poland), aged 19-40 years old participated in the study. Data regarding sociodemographic, family status, dietary and lifestyle characteristics were collected through structured interviews using food frequency questionnaire KomPAN (Jezewska-Zychowicz et al. 2018, http://www.knozc.pan.pl). Dietary and lifestyle patterns were derived using Principal Component Analysis (PCA). In total, 25 dietary and 6 lifestyle standardised variables were included in the PCA. The associations were verified using logistic regression. Four DLPs were derived, explaining $33 \%$ of the variance. Men from the upper tertile of "Protein food, fried-food and recreational physical activity" pattern (characterised by frequent intake of white meat, white rice, eggs, red meat, fried food, groats, higher number of meals a day and recreational activity) were more likely to be under 30 years old (odds ratio, $\mathrm{OR}=2.20,95 \%$ confidence interval, $95 \% \mathrm{CI}$ : $1.30-3.70$ ) than men from the bottom tertile. Men from the upper tertile of "Sandwiches and convenience foods" pattern (characterised by frequent intake of cold meats and smoked sausages, white bread, butter, cheese, sweets and tinned meat) were more likely to be in a relationship $(1.95,1.14-3.34)$ than men from the bottom tertile. Men from the upper tertile of "Fast foods and stimulants" pattern (characterised by frequent intake of sweetened beverages, energy drinks, alcohol, fast foods, and smoking currently or/and in the past) were more likely to be under 30 years old $(2.46,1.46-4.14)$, to live in towns and rural areas $(2.07,1.21-3.55)$ and to have lower educational level $(2.01,1.20-3.36)$ in comparison to men from the bottom tertile. Men from the upper tertile of "Healthy diet, activity at work, former smoking" pattern (characterised by frequent intake of fruit, vegetables, fermented milk products, wholegrain bread, fish, cottage cheese, milk, groats, legumes, higher number of meals a day, physical activity at work and smoking in the past) were more likely to work physically $(2.06,1.22-3.47)$ and be in a relationship $(1.93,1.12-3.32)$. Sociodemographic and family correlates were strongly associated with dietary and lifestyle patterns, both healthy and unhealthy, in our sample of young men. The results demonstrate that diet and lifestyle interventions should be particularly targeted at men under 30 years old, those living in small towns and rural areas and with lower educational level.

\section{Conflict of Interest}

There is no conflict of interest. Project financially supported by Minister of Science and Higher Education in the range of the program entitled "Regional Initiative of Excellence" for the years 2019-2022, Project No. 010/RID/2018/19, amount of funding 12.000.000 PLN. 